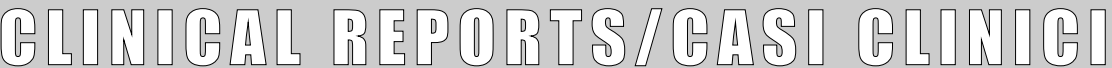

\section{Infestazione sottocutanea da Dirofilaria spp.: descrizione di un caso clinico}

\author{
Ilaria Crespi', Stefano Andreoni', Flavio Bobbio², Antonella Bruno ${ }^{3}$, Roberta Maserati \\ Giacomo Fortina'
}

'Laboratorio di Microbiologia e Virologia, Azienda Ospedaliera Maggiore della Carità, Novara

2II Medicina, Azienda Ospedaliera Maggiore della Carità, Novara

${ }^{3}$ Laboratori di Ricerca Area Infettivologica, Dipartimento di Malattie Infettive, Fondazione IRCCS Policlinico San Matteo, Pavia

Key words: Dirofilariases, filarial nematodes, Dirofilaria spp.

Subcutaneous infection caused by Dirofilaria spp.: a case report

\section{SUMMARY}

Dirofilaria spp. causes a common zoonotic filarial infection found in dogs and humans in the tropical, subtropical and temperate areas. After biological development in the mosquito, dogs and humans may be infected with the infective larvae when the mosquito takes a blood meal. In humans, the worms do not reach maturity, and no microfilariae can be detected. A number of species, such as D. tenuis, D. repens, D. immitis have been identified as causative agents of subcutaneous or conjunctival nodules in humans. Diagnosis is made by identifying the worm in biopsies or extracting the worm from the lesion. Surgical removal of the worms is the only known treatment.

\section{INTRODUZIONE}

Le filarie, naturali parassiti di canidi e felini, sono trasmesse da varie specie di zanzare e possono accidentalmente infestare anche l'uomo; la specie più frequentemente riscontrata nelle nostre zone è Dirofilaria repens (1).

Una volta penetrate nell'insetto in seguito ad assunzione di sangue infetto, le microfilarie evolvono fino allo stadio di larve e possono essere trasmesse ad altri soggetti in occasione di successive punture.

Se il nuovo ospite è "idoneo", si compiranno le mute che porteranno alla formazione di vermi adulti che si localizzano preferibilmente nel miocardio e nell'arteria polmonare per D.immitis e nel tessuto sottocutaneo per le altre specie.

Nel caso dell'uomo, ospite accidentale, la risposta immunitaria del soggetto solitamente blocca lo sviluppo completo del parassita impedendo la formazione di microfilarie nel sangue e conseguentemente l'eventuale trasmissione ad altri soggetti. Le larve in vari stadi di sviluppo e le macrofilarie generalmente portano nell'uomo alla formazione di noduli localizzati nel sottocutaneo o a livello polmonare, oppure in alcuni rari casi le larve possono migrare nel sottocute e a livello congiuntivale (2).

I sintomi riferiti sono aspecifici e i segni clinici più frequenti sono edemi e lesioni ad aspetto nodulare che, se localizzati nel sottocutaneo, aumentano di volume, diventano dolorosi alla palpazione, duri, elastici e circondati da eritema.
Durante l'infestazione non è generalmente presente eosinofilia(3).

Le indagini radiologiche generalmente rilevano noduli; l'ecografia rileva cisti ad aspetto neoplastico, ma l'agoaspirato porta ad una diagnosi definitiva dimostrando la presenza di Dirofilarie.

Segnalazioni di D.immitis provengono da varie parti del mondo, in particolare nel Nord America; di D.repens si hanno riscontri in circa 30 paesi del Vecchio Mondo, Italia compresa (4). Le specie dei generi Aedes, Anopheles e Culex sono ritenute i vettori più idonei per Dirofilaria spp. La recente introduzione anche in Italia di A.albopictus, l'adattabilità di alcune specie di zanzare a differenti condizioni climatiche e a differenti ospiti (uomo e/o carnivori domestici) stanno avendo un ruolo importante nella diffusione dell'infestazione (5).

L'esemplare asportato, alterato dalla risposta immunitaria dell'ospite, è solitamente privo dell'unico carattere morfologico (cuticola esterna) utilizzabile ai fini diagnostici per la differenziazione di specie nelle sezioni istologiche (cuticola liscia in D.immitis, con creste longitudinali nelle altre specie). Attualmente le dirofilarie vengono caratterizzate sotto il profilo biomolecolare distinguendone in questo modo inequivocabilmente la specie (6).

\section{CASO CLINICO}

F.M. anni 33, professione escavatore, da due mesi lamenta un nodulo sottocutaneo in regione sottocostale sulla linea mammaria sinistra. La cute 
sovrastante è lievemente arrossata con aspetto a buccia d'arancia; il paziente riferisce che negli ultimi giorni il nodulo si è ingrandito ed è diventato dolente. Riferisce inoltre di essere stato punto in sede di lavoro durante i mesi estivi da un insetto una decina di $\mathrm{cm}$ più lateralmente rispetto al nodulo attuale e di aver accusato per alcuni giorni un dolore abbastanza intenso nella sede della puntura, seguito successivamente dalla comparsa del nodulo.

Il paziente riferisce anche di aver soggiornato due settimane in Messico, ma assicura che il nodulo era già presente alla partenza per il periodo di vacanza.

Al Dipartimento di Emergenza ed Accettazione (DEA) si esegue ecografia del nodulo (Figura III) e si evidenzia un'immagine anecogena caratterizzata da echi a binario con movimenti spontanei. Si decide quindi di asportare il nodulo con una losanga di cute sovrastante. Il nodulo viene inciso ed all'interno viene evidenziata una struttura filiforme che è stata sottoposta ad esame microscopico diretto (Figura I-II). L'esame istologico ha successivamente confermato che il nodulo cutaneo era costituito da un granuloma con spiccata componente eosinofila.

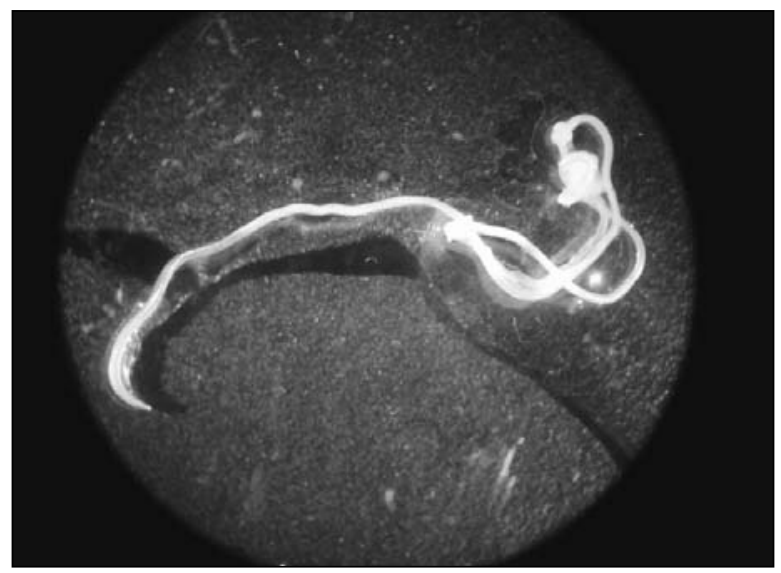

Figura I: Dirofilaria spp.(microscopio ottico)
Una settimana dopo l'escissione chirurgica il paziente si è presentato al controllo ambulatoriale completamente asintomatico.

Gli esami ematochimici, in particolare 1'eosinofilia periferica, erano assolutamente nella norma.

\section{DISCUSSIONE}

L'osservazione microscopica ha evidenziato la presenza di una formazione piatta, bianca, filiforme di circa 25-30 mm di lunghezza convalidando l'ipotesi di infestazione da Dirofilaria spp. Non è stato possibile effettuare una tipizzazione molecolare, unico metodo applicabile per l'identificazione di specie, poiché il reperto era stato conservato in formalina. Nonostante il tentativo di conservazione in formolo, le caratteristiche morfologiche distintive del parassita risultavano parzialmente alterate e quindi non è stato possibile procedere all'identificazione istologica (7). Si ritiene comunque che possa verosimilmente trattarsi di D.repens, essendo questa la specie più diffusa nelle nostre zone e responsabile di formazioni nodulari sottocutanee. La descrizione di questo caso e la revisione di altri simili riportati dalla letteratura nazionale ed internazionale porta a ritenere che la dirofilariosi sia una patologia sottosti-

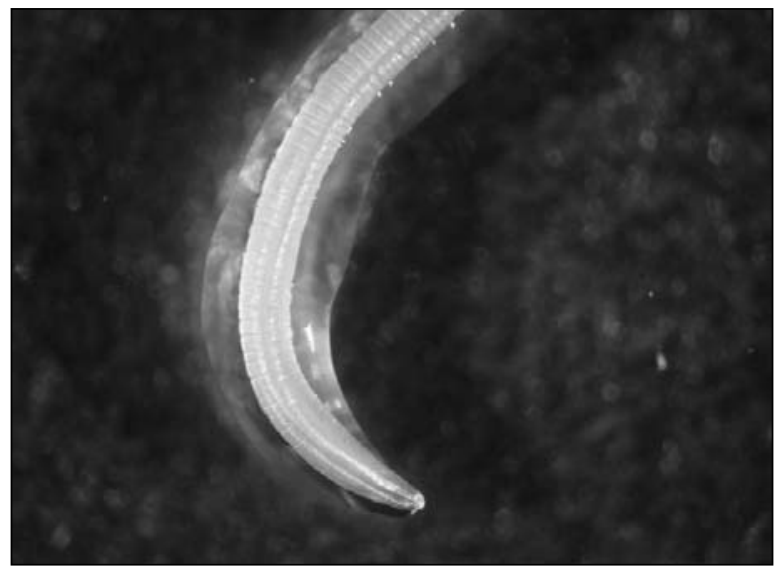

Figura II: particolare di Dirofilaria spp.(microscopio ottico)

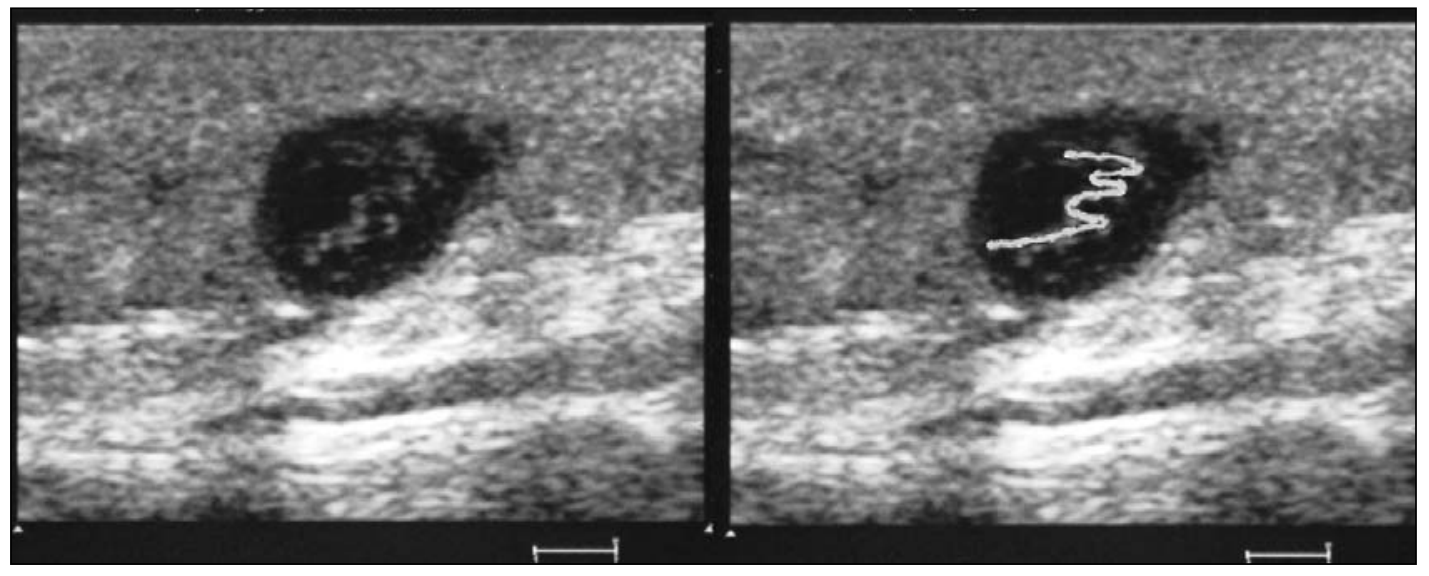

Figura III: ecografia del nodulo sottocutaneo. 
mata, poiché molti sono i casi asintomatici o non diagnosticati e spesso la guarigione avviene spontaneamente, non necessitando di intervento chirurgico che tuttavia rappresenta comunque una terapia risolutiva.

\section{BIBLIOGRAFIA}

1. Kramer L.H., Karteshev V.V., Grandi G., et al. Human subcutaneous dirofilariasisi, Russia. Emerging Infectious Diseases 2006; 13: 150-152.

2. Orihel T.C., Eberhard M.L. Zoonotic filariasis. Clin. Microbiol.Rev.1998; 11: 366-381.

3. Cancrini G., Montoya M.N., Prieto G., BornayLinares F.J. Current advances in experimental diagnosis of human and animal dirofilariosis. In: Heartworm Infection in Human and Animals. 2001; Ediciones Universidad Salamanca,pp. 103-120.

4. Muro A., Genchi C., Cordero M., Simón F. Human dirofilariasis in the European Union. Parasitology Today 1999; 15: 386-389.

5. Pampiglione S., Canestri-Trotti G., Rivasi F. Human dirofilariosis due to Dirofilaria repens: a review of the world literature. Parassitologia 1995; 37: 149-193.

6. Bernieri F., Crotti D., Galli D., Raglio A. Manuale illustrato di diagnostica parassitologica, Selecta Medica Ed. 2001.

7. Cancrini G. Mansonellosi e filariosi zoonotiche. In: Scaglia M., Gatti S., Rondanelli E.G. Parassiti e Parassitosi umane. Dalla clinica al laboratorio. Selecta Medica; 2006; pp. 365-375.

\section{Ilaria Crespi}

Laboratorio di Microbiologia e Virologia, Azienda Ospedaliera Maggiore della Carità Corso Mazzini, 18 - 28100 Novara Tel.: 03213733595 - Fax: 03213733588 E-mail: salmonella@maggioreosp.novara.it 